\title{
Coronavirus Disease (COVID-19) Outbreak: Hypofractionated Radiotherapy in Soft Tissue Sarcomas as a Valuable Option in the Environment of Limited Medical Resources and Demands for Increased Protection of Patients
}

\begin{abstract}
Mateusz Jacek Spałek* and Piotr Rutkowski
Department of Soft Tissue/Bone Sarcoma and Melanoma, Maria Sklodowska-Curie National Research Institute of Oncology, Warsaw, Poland
\end{abstract}

Keywords: sarcoma, hypofractionation, perioperative radiotherapy, COVID-19, multidisciplinary treatment, rare cancers

\section{OPEN ACCESS}

Edited by:

Milly Buwenge,

University of Bologna, Italy

Reviewed by:

Francesco Dionisi,

The Trento Proton Therapy

Centre, Italy

Alessio G. Morganti, University of Bologna, Italy

*Correspondence:

Mateusz Jacek Spałek mateusz.spalek@pib-nio.pl

Specialty section

This article was submitted to

Radiation Oncology,

a section of the journal

Frontiers in Oncology

Received: 11 April 2020

Accepted: 19 May 2020

Published: 05 June 2020

Citation:

Spałek MJ and Rutkowski P (2020)

Coronavirus Disease (COVID-19)

Outbreak: Hypofractionated Radiotherapy in Soft Tissue Sarcomas

as a Valuable Option in the Environment of Limited Medical

Resources and Demands for

Increased Protection of Patients.

Front. Oncol. 10:993.

doi: 10.3389/fonc.2020.00993

\section{INTRODUCTION}

The coronavirus disease (COVID-19) pandemic in 2019/2020 became a significant problem not only for first-line healthcare but also for cancer patients, who are at the risk of severe or fatal outcome of potential infection. In recently published papers about radiotherapy (RT) in various cancers, there are suggestions to use more hypofractionated RT (HFRT) regimens during the COVID-19 pandemic to reduce overall treatment time (1). Unfortunately, HFRT in soft tissue sarcomas (STS) is rarely mentioned and underestimated. SARS-CoV-2 infection in a tertiary STS clinic or RT department might cause a shortage of experienced staff by putting them in quarantine. What is more, the interrupted treatment cannot be continued in other institutions with the maintenance of high-quality care due to lack of necessary knowledge, experience, and equipment. Perioperative conventionally fractionated RT (CFRT), namely between 1.8 and 2.0 Gy per fraction by 5 to 7 weeks, is considered to be a standard regimen in STS (2). There is growing evidence that preoperative HFRT could be also a possible therapeutic approach and its wide introduction may be a controversial but necessary solution.

\section{RATIONALE FOR PREOPERATIVE APPROACH}

Preoperative RT in STS is not widely accepted due to the higher risk of postoperative wound complications. However, wound complications, even serious, are usually manageable and reversible, while late toxicity, manifested as complications related to fibrosis, is commonly permanent and can lead to severe impairment of patient's function and quality of life. In a phase III randomized clinical trial that compared preoperative and postoperative RT in STS, wound complications occurred in 35\% of patients in the preoperative group and in $17 \%$ in the postoperative group (3). After prolonged follow-up, late toxicity was observed more frequently in the postoperative arm than in the preoperative arm without any significant differences in local control and survival (4). The preoperative RT has more advantages i.e., visible tumor volume, less healthy tissues within irradiated volume, lower total dose, better tissue oxygenation, and lower risk of tumor cell seeding during surgery. Moreover, preoperative RT may provide substantial benefit for patients with locally advanced disease, allowing conservative or limb-sparing surgery in 
marginally-resectable or unresectable STS (5). Finally, costeffectiveness analysis supports preoperative RT in STS (6).

\section{RATIONALE FOR HYPOFRACTIONATION}

HFRT has a clinical rationale. HFRT regimens could significantly shorten overall treatment time. Decreased exposure to potential SARS-CoV-2 infection in a hospital as well as compliance with treatment, convenience and cost favor HFRT. Additionally, HFRT has a radiobiological rationale. Basing on the linearquadratic model, a larger dose per fraction applied to tumors with a lower $\alpha / \beta$ ratio should result in better tumor control. Heterogeneity of STS translates into a wide spectrum of radiosensitivity, however, for most STS subtypes $\alpha / \beta$ ratio is considered as lower than $10 \mathrm{~Gy}$ (7). For example, calculated liposarcoma and rhabdomyosarcoma $\alpha / \beta$ ratios were as low as 0.4 and 2.8 Gy, respectively (8). Furthermore, assuming low $\alpha / \beta$ ratio for STS and better responsiveness to a larger fraction size, HFRT may allow de-escalation of total dose with constant tumor control. That may result in decreased toxicity from surrounding tissues. For purposes of comparison of the different fractionation schedules in this review, the equivalent dose in 2-Gy fractions (EQD2) was calculated assuming the $\alpha / \beta$ ratio for STS of $4 \mathrm{~Gy}$, as in calculations performed in other studies $(2,7)$. The results were presented in Table $\mathbf{1}$.

\section{AVAILABLE EVIDENCE AND HFRT REGIMENS}

Preoperative HFRT in STS has been validated in retrospective analyses, prospective registries, and phase I-II clinical trials (5, 23-31). However, randomized phase III trials comparing preoperative CFRT with HFRT are lacking. As with CFRT, HFRT could be combined with systemic treatment (2). Despite scarce evidence on the efficacy of perioperative chemotherapy in STS, it is commonly applied as a part of treatment. A combination of RT with targeted therapy is still under investigation giving promising results but also unexpected toxicities $(18,21)$. The investigated regimens of preoperative RT in STS were summarized in Table 1 $(3-5,9-31)$. Presented data should be interpreted with caution because analyzed populations were not comparable as they differed with many factors including patients' characteristics, STS subtypes, tumor size, indications for RT, RT techniques, elective margins, and quality of surgery. Nevertheless, the results of HFRT regimens seem very similar to those of CFRT regimens. The 5year local control was $82-100 \%$ (median 91\%) in CFRT and 89 and $97 \%$ in two studies on HFRT. Furthermore, the rate of severe wound complications was $0-25 \%$ (median 17\%) in CFRT and 1$24 \%$ (median 18\%) in HFRT. It is noticeable, that EQD2 is lower than $50 \mathrm{~Gy}$ in the majority of analyzed HFRT regimens.

\section{DISCUSSION}

Available data suggest that preoperative HFRT in STS is a promising treatment option providing satisfactory local control with acceptable toxicity. Nevertheless, it has been not widely adapted in clinical practice. COVID-19 pandemic may be the appropriate time to rethink RT in STS.

Routine use of preoperative HFRT may be limited by some concerns. One may fear that decreased EQD2 in preoperative HFRT will result in worse local control. However, the current standard of $50 \mathrm{~Gy}$ in 2-Gy fractions is not based upon strong evidence coming from randomized clinical trials with various dose levels or fractionation regimens. In the analysis performed by Haas et al. it has been shown that dose-response relationship for local control in preoperative RT is clear only below $28 \mathrm{~Gy}$ in 8 fractions of $3.5 \mathrm{~Gy}(\mathrm{EQD} 2=35 \mathrm{~Gy}$ if $\alpha / \beta=4 \mathrm{~Gy})$ (2). Above that level, the benefit in local control from increased total dose may be negligible, especially when RT is combined with preoperative chemotherapy or targeted therapy. Data presented in the Table 1 suggest that this assumption may be correct because local control in all described regimens is higher than $80 \%$ despite various EQD2. Interestingly, HFRT regimen described by Koseła-Paterczyk et al. (25 Gy in 5 fractions) given in the majority of patients without preoperative chemotherapy provided lower (but acceptable) local control than regimens with higher fraction and total doses (30 Gy in 5 fractions) or one with the same fractionation regimen but combined with sequential anthracycline-based chemotherapy $(5,27,30$, $31)$. The same $5 \times 5$ Gy regimen without chemotherapy but with delayed surgery resulted in 100\% 1-year control rate in patients with myxoid liposarcomas that are considered radiosensitive (29).

Furthermore, the preoperative approach and hypofractionation in STS remain controversial due to the risk of treatment-related morbidity. Wound complications are serious adverse effects of any preoperative RT in STS. However, this toxicity could be predicted by assessment of patient-related risk factors, such as smoking, diabetes, obesity, and tumor location (lower limbs) (32). Although larger doses per fraction could theoretically increase the risk of late toxicities, such assumption was neither confirmed in clinical trials with preoperative and definitive HFRT in other neoplasms, i.e., rectal, prostate or lung cancer, nor in presented data regarding HFRT in STS. Moreover, the occurrence of selected late toxicities after combined treatment of STS could be predicted and often reduced. For example, periosteal location of tumor, higher mean and maximal dose to bone as well as volume of bone irradiated to over $40 \mathrm{~Gy}$ in 2-Gy fractions increase the risk of pathologic fractures (33). Proper treatment planning and choice of RT techniques with intensity modulation can significantly reduce both early and late toxicity $(17,20)$. Thus, taking into account local control and toxicity, the choice of RT regimen should be based on several factors, i.e., patients' characteristics, tumor location and size, STS subtype and its radiosensitivity, risk of local and distant relapse, availability of equipment, RT techniques, and systemic treatments.

No direct comparison of preoperative CFRT and HFRT regimens in STS was performed in the literature. While randomized clinical trials are still the gold standard, other approaches when investigating various treatments for rare diseases should be considered, such as Bayesian trial design. Moreover, after discussion within the multidisciplinary tumor board, the individualized treatment 
TABLE 1 | Preoperative radiotherapy regimens in soft tissue sarcomas in major published studies.

\begin{tabular}{|c|c|c|c|c|c|c|c|c|c|c|c|c|}
\hline & References & Evidence & $\mathrm{N}$ of patients & $\begin{array}{l}\text { Dominant } \\
\text { preoperative } \\
\text { regimen }\end{array}$ & $\begin{array}{c}\text { EQD2 } \\
\alpha / \beta \\
4 \text { Gy }\end{array}$ & $\begin{array}{l}\text { Tumors } \\
>10 \mathrm{~cm}\end{array}$ & $\begin{array}{l}\text { Surgery } \\
\text { after RT }\end{array}$ & Ro & $\begin{array}{l}\text { @years } \\
\text { local control }\end{array}$ & $\begin{array}{l}\text { All wound } \\
\text { complications } \\
@ \text { severe }^{\wedge}\end{array}$ & $\begin{array}{l}\text { Reported } \\
\text { late toxicity }\end{array}$ & $\begin{array}{c}\text { @years } \\
\text { estimated } \\
\text { survival }\end{array}$ \\
\hline \multirow[t]{10}{*}{$\begin{array}{l}\text { Conventionally } \\
\text { fractionated } \\
\text { RT regimens }\end{array}$} & Pollack et al. (9) & $\begin{array}{l}\text { Retrospective } \\
\text { cohort }\end{array}$ & $\begin{array}{c}128 \text { (preop) } \\
51 \mathrm{CHT}+\mathrm{RT} \\
77 \mathrm{RT}\end{array}$ & $\begin{array}{c}\mathrm{CHT}^{*} \& \\
50 \mathrm{~Gy} / 25 \mathrm{fr} .\end{array}$ & 50 Gy & $\begin{array}{l}\text { ND } \\
\text { median } \\
10 \mathrm{~cm}\end{array}$ & Delayed & $92 \%$ & $\begin{array}{l}@ 5 y \\
82 \%\end{array}$ & 25\%@ND & $6 \%$ & ND \\
\hline & $\begin{array}{l}\text { O'Sullivan et al. } \\
(3,10) \\
\text { Davis et al. (4) }\end{array}$ & $\begin{array}{l}\text { Phase III RCT } \\
\text { (preop vs. } \\
\text { postop) }\end{array}$ & 94 (preop) & 50 Gy/25 fr. & 50 Gy & $35 \%$ & $\begin{array}{c}\text { Delayed } \\
\text { (3-6 weeks) }\end{array}$ & $84 \%$ & $\begin{array}{l}@ 5 y \\
93 \%\end{array}$ & 35\%@17\% & $\begin{array}{c}\text { G2+: } \\
\text { fibrosis 32\% } \\
\text { JS 18\% } \\
\text { edema 15\% }\end{array}$ & $\begin{array}{c}\text { @5y } \\
\text { DRFS 67\% } \\
\text { OS 73\% }\end{array}$ \\
\hline & Zagars et al. (11) & $\begin{array}{l}\text { Retrospective } \\
\text { cohort }\end{array}$ & $\begin{array}{c}271 \text { (preop) } \\
179 \mathrm{CHT}+\mathrm{RT} \\
92 \mathrm{RT}\end{array}$ & $\begin{array}{c}\mathrm{CHT}^{*} \& \\
50 \mathrm{~Gy} / 25 \mathrm{fr} .\end{array}$ & 50 Gy & $42 \%$ & $\begin{array}{l}\text { Delayed } \\
\text { (4-6 weeks) }\end{array}$ & $86 \%$ & $\begin{array}{l}@ 5 y \\
85 \% \\
@ 10 y \\
83 \%\end{array}$ & ND & $5 \%$ & $\begin{array}{c}@ 5 y \\
\text { DRFS 64\% } \\
\text { @10y } \\
\text { DRFS 61\% } \\
\text { DSS 64\% }\end{array}$ \\
\hline & Hui et al. (12) & $\begin{array}{l}\text { Retrospective } \\
\text { cohort }\end{array}$ & 67 & 50.4 Gy/28 fr. & 48.7 Gy & $\begin{array}{l}\mathrm{ND} \\
\text { median } \\
6 \mathrm{~cm}\end{array}$ & $\begin{array}{c}\text { Delayed } \\
\text { (3-6 weeks) }\end{array}$ & $99 \%$ & $\begin{array}{l}@ 5 y \\
93 \%\end{array}$ & 41\%@18\% & $7 \%$ & $\begin{array}{c}\text { @5y } \\
\text { DRFS 68\% } \\
\text { OS 73\% }\end{array}$ \\
\hline & Kraybill et al. (13) & $\begin{array}{l}\text { Phase II } \\
\text { single arm CT }\end{array}$ & 64 & $\begin{array}{c}\text { MAID } \\
22 \text { Gy/11 fr. } \\
\text { MAID } \\
22 \text { Gy/11 fr. } \\
\text { MAID }\end{array}$ & 44 Gy & $\begin{array}{c}N D \\
\text { median } \\
15 \mathrm{~cm}\end{array}$ & Delayed & $91 \%$ & $\begin{array}{l}@ 3 y \\
90 \%\end{array}$ & 11\%@3\% & ND & $\begin{array}{c}@ 3 y \\
\text { DRFS 65\% } \\
\text { OS 75\% }\end{array}$ \\
\hline & Canter et al. (14) & $\begin{array}{l}\text { Retrospective } \\
\text { cohort }\end{array}$ & 25 & 50 Gy/25 fr. & 50 Gy & $36 \%$ & $\begin{array}{c}\text { Delayed } \\
\text { (4-6 weeks) }\end{array}$ & $84 \%$ & $\begin{array}{l}@ 3 y \\
100 \%\end{array}$ & 28\%@16\% & ND & ND \\
\hline & Yoon et al. (15) & $\begin{array}{l}\text { Phase II } \\
\text { single arm CT }\end{array}$ & 20 & $\begin{array}{l}\text { Bevacizumab } \\
50.4 \text { Gy/28 fr. }\end{array}$ & 48.7 Gy & $\begin{array}{l}\mathrm{ND} \\
\text { median } \\
8 \mathrm{~cm}\end{array}$ & $\begin{array}{l}\text { Delayed } \\
\text { (6-7 weeks) }\end{array}$ & ND & $\begin{array}{l}@ 2 y \\
95 \%\end{array}$ & 20\%@ND & ND & $\begin{array}{c}\text { @2y } \\
\text { DRFS 65\% }\end{array}$ \\
\hline & Shah et al. (16) & $\begin{array}{l}\text { Retrospective } \\
\text { cohort }\end{array}$ & 30 & 50 Gy/25 fr. & 50 Gy & $40 \%$ & $\begin{array}{l}\text { Delayed } \\
\text { (4-6 weeks) }\end{array}$ & ND & $\begin{array}{l}@ 5 y \\
100 \%\end{array}$ & 23\%@20\% & ND & $\begin{array}{c}\text { @5y } \\
\text { DRFS 61\% } \\
\text { OS 69\% }\end{array}$ \\
\hline & $\begin{array}{l}\text { O'Sullivan et al. } \\
\text { (17) }\end{array}$ & $\begin{array}{l}\text { Phase II } \\
\text { single arm CT }\end{array}$ & 59 & 50 Gy/25 fr. & 50 Gy & $\begin{array}{l}\mathrm{ND} \\
\text { median } 10 \mathrm{~cm}\end{array}$ & Delayed & $93 \%$ & $\begin{array}{l}@ 5 y \\
88 \%\end{array}$ & 31\%@10\% & $\begin{array}{c}\text { Moderate: } \\
\text { skin 2\% } \\
\text { fibrosis 9\% } \\
\text { JS 7\% } \\
\text { edema 11\% }\end{array}$ & $\begin{array}{c}\text { @5y } \\
\text { DRFS 67\% } \\
\text { OS 75\% }\end{array}$ \\
\hline & Lewin et al. (18) & $\begin{array}{l}\text { Phase Ib/II } \\
\text { single arm CT }\end{array}$ & 9 & $\begin{array}{l}\text { Sunitinib } \\
50.4 \text { Gy/28 fr. }\end{array}$ & 48.7 Gy & $\begin{array}{l}\text { ND } \\
\text { median } \\
10 \mathrm{~cm}\end{array}$ & $\begin{array}{c}\text { Delayed } \\
\text { (3-6 weeks) }\end{array}$ & ND & ND & ND & Any G: 78\% & $\begin{array}{c}\text { @2y } \\
\text { PFS 44\% } \\
\text { OS 56\% }\end{array}$ \\
\hline
\end{tabular}


TABLE 1 | Continued

\begin{tabular}{|c|c|c|c|c|c|c|c|c|c|c|c|c|}
\hline & References & Evidence & $\mathrm{N}$ of patients & $\begin{array}{l}\text { Dominant } \\
\text { preoperative } \\
\text { regimen }\end{array}$ & $\begin{array}{l}\text { EQD2 } \\
\alpha / \beta \\
4 \text { Gy }\end{array}$ & $\begin{array}{l}\text { Tumors } \\
>10 \mathrm{~cm}\end{array}$ & $\begin{array}{l}\text { Surgery } \\
\text { after RT }\end{array}$ & Ro & $\begin{array}{l}\text { @years } \\
\text { local control }\end{array}$ & $\begin{array}{c}\text { All wound } \\
\text { complications } \\
\text { @severe }\end{array}$ & $\begin{array}{l}\text { Reported late } \\
\text { toxicity }\end{array}$ & $\begin{array}{c}\text { @years } \\
\text { estimated } \\
\text { survival }\end{array}$ \\
\hline & Canter et al. (19) & $\begin{array}{l}\text { Phase I single } \\
\text { arm CT }\end{array}$ & 8 & $\begin{array}{l}\text { Sorafenib } \\
50 \text { Gy/25 fr. }\end{array}$ & 50 Gy & $63 \%$ & $\begin{array}{l}\text { Delayed } \\
\text { (4-6 weeks) }\end{array}$ & $75 \%$ & $\begin{array}{l}@ 3 y \\
100 \%\end{array}$ & 38\%@ND & ND & $\begin{array}{c}\text { @ } 3 y \\
\text { DRFS 42\% } \\
\text { OS 75\% }\end{array}$ \\
\hline & Wang et al. (20) & $\begin{array}{l}\text { Phase II } \\
\text { single arm CT }\end{array}$ & 79 & $\begin{array}{c}50 \text { Gy/25 fr. } \\
\text { with } \\
\text { reduced margins }\end{array}$ & 50 Gy & $\begin{array}{c}\mathrm{ND} \\
\text { median } \\
11 \mathrm{~cm}\end{array}$ & Delayed & $76 \%$ & $\begin{array}{l}@ 2 y \\
94 \%\end{array}$ & 37\%@25\% & $\begin{array}{c}\text { G2+: } \\
\text { fibrosis 5\% } \\
\text { JS 4\% } \\
\text { edema 5\% }\end{array}$ & $\begin{array}{c}\text { @2y } \\
\text { DRFS 65\% } \\
\text { OS 81\% }\end{array}$ \\
\hline & Haas et al. (21) & $\begin{array}{l}\text { Phase I single } \\
\text { arm CT }\end{array}$ & 11 & $\begin{array}{l}\text { Pazopanib } \\
50 \text { Gy/25 fr. }\end{array}$ & 50 Gy & $27 \%$ & $\begin{array}{l}\text { Delayed } \\
\text { (5-7 weeks) }\end{array}$ & ND & $\begin{array}{l}@ 2 y \\
91 \%\end{array}$ & 20\%@0\% & ND & $\begin{array}{c}\text { @2y } \\
\text { DRFS 82\% }\end{array}$ \\
\hline & Jakob et al. (22) & $\begin{array}{l}\text { Phase } \mathrm{lb} / \mathrm{ll} \\
\text { single arm CT }\end{array}$ & 5 & $\begin{array}{l}\text { Sunitinib } \\
50.4 \text { Gy/28 fr. }\end{array}$ & 48.7 Gy & $40 \%$ & $\begin{array}{l}\text { Delayed } \\
\text { (5-8 weeks) }\end{array}$ & $100 \%$ & $\begin{array}{l}@ 2 y \\
80 \%\end{array}$ & 56\%@22\% & ND & $\begin{array}{c}\text { @2y } \\
\text { DRFS 60\% }\end{array}$ \\
\hline \multirow[t]{6}{*}{$\begin{array}{l}\text { Hypofractionated } \\
\text { RT regimens }\end{array}$} & Temple et al. (23) & $\begin{array}{l}\text { Prospective } \\
\text { register }\end{array}$ & 42 & $\begin{array}{l}\text { Doxorubicin } \\
30 \text { Gy/10 fr. }\end{array}$ & 35 Gy & ND & $\begin{array}{l}\text { Delayed } \\
\text { (4-6 weeks) }\end{array}$ & ND & $\begin{array}{l}@ 5 y \\
97 \%\end{array}$ & 15\%@ND & ND & $\begin{array}{c}\text { @5y } \\
\text { OS 79\% }\end{array}$ \\
\hline & Ryan et al. (24) & $\begin{array}{l}\text { Retrospective } \\
\text { cohort }\end{array}$ & 25 & $\begin{array}{c}\text { El } \\
28 \mathrm{~Gy} / 8 \mathrm{fr} .\end{array}$ & $35 \mathrm{~Gy}$ & $\begin{array}{c}N D \\
\text { median } \\
10 \mathrm{~cm}\end{array}$ & $\begin{array}{c}\text { Delayed } \\
\text { (4-5 weeks) }\end{array}$ & $88 \%$ & $\begin{array}{l}@ 2 y \\
88 \%\end{array}$ & ND@20\% & ND & $\begin{array}{c}\text { @2y } \\
\text { DRFS 78\% } \\
\text { OS 84\% }\end{array}$ \\
\hline & $\begin{array}{l}\text { MacDermed et al. } \\
\text { (25) }\end{array}$ & $\begin{array}{l}\text { Retrospective } \\
\text { cohort }\end{array}$ & $\begin{array}{c}34 \\
\text { included } 6 \\
\text { patients } \\
\text { with DM }\end{array}$ & $\begin{array}{l}\text { Ifosfamide } \\
28 \mathrm{~Gy} / 8 \mathrm{fr} \text {. }\end{array}$ & 35 Gy & $\begin{array}{c}32 \% \\
(>12 \mathrm{~cm})\end{array}$ & $\begin{array}{l}\text { delayed } \\
\text { (4-8 weeks) }\end{array}$ & $100 \%$ & $\begin{array}{l}@ 5 y \\
89 \%\end{array}$ & ND@17\% & $\begin{array}{l}\text { Fibrosis } 14 \% \\
\text { edema } 17 \%\end{array}$ & $\begin{array}{c}\text { @5y (no } \\
\text { DM) } \\
\text { DRFS 53\% } \\
\text { OS 45\% }\end{array}$ \\
\hline & Meyer et al. (26) & $\begin{array}{l}\text { Phase I single } \\
\text { arm CT }\end{array}$ & $\begin{array}{c}16 \\
\text { included } 2 \\
\text { patients } \\
\text { with DM }\end{array}$ & $\begin{array}{l}\text { Sorafenib } \\
\text { El } \\
28 \mathrm{~Gy} / 8 \mathrm{fr} .\end{array}$ & 35 Gy & ND & Delayed & $94 \%$ & $\begin{array}{l}@ 2 y \\
100 \%\end{array}$ & 38\%@ND & ND & $\begin{array}{c}\text { @2y } \\
\text { PFS 86\% }\end{array}$ \\
\hline & $\begin{array}{l}\text { Kosela-Paterczyk } \\
\text { et al. (27) }\end{array}$ & $\begin{array}{l}\text { Prospective } \\
\text { register }\end{array}$ & $\begin{array}{c}272 \\
61 \mathrm{CHT}+\mathrm{RT} \\
211 \mathrm{RT}\end{array}$ & $\begin{array}{c}\mathrm{CHT}^{*} \& \\
25 \mathrm{~Gy} / 5 \mathrm{fr} .\end{array}$ & 37.5 Gy & $42 \%$ & $\begin{array}{l}\text { Immediate } \\
\text { (3-7 days) }\end{array}$ & $79 \%$ & $\begin{array}{l}@ 3 y \\
81 \%\end{array}$ & $\begin{array}{c}\text { all } 32 \% @ 12 \% \\
53 \% \text { CHT+RT } \\
53 \% @ 21 \% \\
\text { RT } 27 \% @ 9 \%\end{array}$ & $\begin{array}{c}15 \% \text { all } \\
23 \% \text { CHT+RT } \\
12 \% \text { RT }\end{array}$ & $\begin{array}{l}\text { @5y } \\
\text { OS 60\% }\end{array}$ \\
\hline & $\begin{array}{l}\text { Pennington et al. } \\
\text { (28) }\end{array}$ & $\begin{array}{l}\text { Retrospective } \\
\text { cohort }\end{array}$ & 116 & $\begin{array}{c}\mathrm{CHT}^{\star} \\
28 \mathrm{~Gy} / 8 \mathrm{fr} .\end{array}$ & 35 Gy & $47 \%$ & $\begin{array}{l}\text { Delayed } \\
\text { (2-3 weeks) }\end{array}$ & $93 \%$ & $\begin{array}{l}@ 3 y \\
89 \% \\
@ 6 y \\
83 \%\end{array}$ & 10\%@1\% & $4 \%$ & $\begin{array}{c}@ 3 y \\
\text { DRFS 75\% } \\
\text { OS 82\% } \\
@ 6 y \\
\text { DRFS 65\% } \\
\text { OS 67\% }\end{array}$ \\
\hline
\end{tabular}


TABLE 1 | Continued

\begin{tabular}{|c|c|c|c|c|c|c|c|c|c|c|c|}
\hline References & Evidence & $\mathrm{N}$ of patients & $\begin{array}{l}\text { Dominant } \\
\text { preoperative } \\
\text { regimen }\end{array}$ & $\begin{array}{c}\text { EQD2 } \\
\alpha / \beta \\
4 \mathrm{~Gy}\end{array}$ & $\begin{array}{l}\text { Tumors } \\
>10 \mathrm{~cm}\end{array}$ & $\begin{array}{l}\text { Surgery } \\
\text { after RT }\end{array}$ & Ro & $\begin{array}{c}\text { @years } \\
\text { local control }\end{array}$ & $\begin{array}{l}\text { All wound } \\
\text { complications } \\
\text { @severe^ }\end{array}$ & $\begin{array}{l}\text { Reported } \\
\text { late toxicity }\end{array}$ & $\begin{array}{c}\text { @years } \\
\text { estimated } \\
\text { survival }\end{array}$ \\
\hline Spalek et al. (29) & $\begin{array}{l}\text { Phase II } \\
\text { single arm CT }\end{array}$ & $\begin{array}{l}29 \\
\text { MLPS only }\end{array}$ & 25 Gy $/ 5$ fr. & $37.5 \mathrm{~Gy}$ & $66 \%$ & $\begin{array}{c}\text { Delayed } \\
\text { (6-8 weeks) }\end{array}$ & $93 \%$ & $\begin{array}{l}@ 1 y \\
100 \%\end{array}$ & 31\%@ND & ND & $\begin{array}{c}@ 1 y \\
\text { DRFS 86\% }\end{array}$ \\
\hline Spalek et al. (5) & $\begin{array}{l}\text { Phase II } \\
\text { single arm CT }\end{array}$ & $\begin{array}{c}30 \\
\text { marginally } \\
\text { resectable } \\
\text { or unresectable }\end{array}$ & $\begin{array}{c}1 \times \mathrm{Al} \\
25 \mathrm{~Gy} / 5 \mathrm{fr} . \\
2 \times \mathrm{Al}\end{array}$ & $37.5 \mathrm{~Gy}$ & $74 \%$ & $\begin{array}{c}\text { Delayed } \\
(6-8 \text { weeks })\end{array}$ & $73 \%$ & $\begin{array}{l}@ 1 y \\
97 \%\end{array}$ & 23\%@7\% & ND & $\begin{array}{c}\text { @1y } \\
\text { DRFS 74\% }\end{array}$ \\
\hline Parsai et al. (30) & $\begin{array}{l}\text { Retrospective } \\
\text { cohort }\end{array}$ & $\begin{array}{c}16 \\
3 \mathrm{CHT}+\mathrm{RT} \\
13 \mathrm{RT}\end{array}$ & $\begin{array}{c}\mathrm{CHT}^{*} \\
30 \mathrm{~Gy} / 5 \mathrm{fr} .\end{array}$ & 50 Gy & $25 \%$ & $\begin{array}{l}\text { Immediate } \\
\text { (0-7 days) }\end{array}$ & $63 \%$ & $\begin{array}{l}@ 1 y \\
100 \%\end{array}$ & 31\%@19\% & ND & ND \\
\hline Kalbasi et al. (31) & $\begin{array}{l}\text { Phase II } \\
\text { single arm CT }\end{array}$ & 50 & 30 Gy/5 fr. & 50 Gy & $24 \%$ & $\begin{array}{l}\text { Delayed } \\
(2-6 \text { weeks })\end{array}$ & $82 \%$ & @2y 94\% & 32\%@24\% & $\begin{array}{c}\text { G1: } \\
\text { fibrosis } 24 \% \\
\text { JS } 11 \% \\
\text { edema } 4 \% \\
\text { G2: } \\
\text { fibrosis } 11 \% \\
\text { JS 11\% } \\
\text { edema 4\% }\end{array}$ & $\begin{array}{c}@ 2 y \\
\text { DRFS 79\% }\end{array}$ \\
\hline
\end{tabular}

Al, doxorubicin, ifosfamide: El epirubicin, ifosfamide: CHT, chemotherapy: CT, clinical trial: DM, distant metastases: DRFS, distant recurrence-free survival; DSS, disease-specific survival: EQD2, equivalent dose in 2-Gy fractions; G, grade; JS, joint stiffness; MAID, mesna, doxorubicin, ifosfamide, dacarbazine; MLPS, myxoid liposarcomas; ND, no data; OS, overall survival; PFS, progression-free survival; RCT, randomized clinical trial; RT, radiotherapy; STS, soft tissue sarcomas.

*various regimens were used.

\& only part of a group received chemotherapy.

$\wedge$ assessed by authors as grade 3 or higher, or requiring reoperation. 
regimens may be proposed to patients then collected in prospective registries.

Radiation oncologists are not front-line fighters in COVID19 times, but they can deal with the spread of infection another way. In a global emerging situation of COVID-19 pandemic, the benefits of preoperative HFRT for STS patients may outweigh risks. Besides good efficacy and acceptable toxicity, HFRT decreases the hospital-associated COVID-19 infection risk, as well as the risk of treatment interruption, delay, or its poor quality if performed outside STS tertiary center. Available treatment options and concerns should be discussed with the patient in a shared decision-making process.

\section{REFERENCES}

1. Achard V, Tsoutsou P, Zilli T. Radiotherapy in the time of the Coronavirus pandemic: when less is better. Int J Radiation Oncol Biol Phys. (2020). doi: 10.1016/j.ijrobp.2020.03.008. [Epub ahead of print].

2. Haas RLM, Miah AB, LePechoux C, DeLaney TF, Baldini EH, Alektiar $\mathrm{K}$, et al. Preoperative radiotherapy for extremity soft tissue sarcoma; past, present and future perspectives on dose fractionation regimens and combined modality strategies. Radiother Oncol. (2016) 119:14-21. doi: 10.1016/j.radonc.2015.12.002

3. O'Sullivan B, Davis AM, Turcotte R, Bell R, Catton C, Chabot P, et al. Preoperative versus postoperative radiotherapy in soft-tissue sarcoma of the limbs: a randomised trial. Lancet. (2002) 359:2235-41. doi: 10.1016/S0140-6736(02)09292-9

4. Davis AM, O'Sullivan B, Turcotte R, Bell R, Catton C, Chabot P, et al. Late radiation morbidity following randomization to preoperative versus postoperative radiotherapy in extremity soft tissue sarcoma. Radiother Oncol. (2005) 75:48-53. doi: 10.1016/j.radonc.2004.12.020

5. Spalek M, Koseła-Paterczyk H, Borkowska A, Wagrodzki M, SzumeraCiećkiewicz A, Cieszanowski A, et al. OC-0069 5x5 Gy with chemotherapy in borderline resectable soft tissue sarcomas: early results of a trial. Radiotherapy and Oncology. (2019) 133:S31-S32. doi: 10.1016/S0167-8140(19)30489-X

6. Qu XM, Louie AV, Ashman J, Wasif N. Cost-Effectiveness Analysis of Preoperative Versus Postoperative Radiation Therapy in Extremity Soft Tissue Sarcoma. Int J Radiat Oncol Biol Phys. (2017) 97:339-346. doi: 10.1016/j.ijrobp.2016.10.009

7. Thames HD, Suit HD. Tumor radioresponsiveness versus fractionation sensitivity. Int J Radiat Oncol Biol Phys. (1986) 12:687-691. doi: 10.1016/0360-3016(86)90081-7

8. van Leeuwen CM, Oei AL, Crezee J, Bel A, Franken N a. P, Stalpers LJA, et al. The alfa and beta of tumours: a review of parameters of the linear-quadratic model, derived from clinical radiotherapy studies. Radiat Oncol. (2018) 13:96. doi: 10.1186/s13014-018-1040-Z

9. Pollack A, Zagars GK, Goswitz MS, Pollock RA, Feig BW, Pisters PW. Preoperative vs. postoperative radiotherapy in the treatment of soft tissue sarcomas: a matter of presentation. Int J Radiat Oncol Biol Phys. (1998) 42:563-72. doi: 10.1016/S0360-3016(98)00277-6

10. O'Sullivan B, Davis A, Turcotte R, Bell R, Wunder J, Catton C, et al. Five-year results of a randomized phase III trial of pre-operative vs postoperative radiotherapy in extremity soft tissue sarcoma. JCO. (2004) 22:9007. doi: 10.1200/jco.2004.22.14_suppl.9007

11. Zagars GK, Ballo MT, Pisters PWT, Pollock RE, Patel SR, Benjamin RS. Preoperative vs. postoperative radiation therapy for soft tissue sarcoma: a retrospective comparative evaluation of disease outcome. Int J Radiat Oncol Biol Phys. (2003) 56:482-8. doi: 10.1016/S0360-3016(02) 04510-8

12. Hui AC, Ngan SY, Wong K, Powell G, Choong PFM. Preoperative radiotherapy for soft tissue sarcoma: the Peter MacCallum Cancer Centre experience. Eur J Surg Oncol. (2006) 32:1159-64. doi: 10.1016/j.ejso.2006.04.003

\section{AUTHOR CONTRIBUTIONS}

MS and PR conceived of the presented idea and discussed the concerns and contributed to the final manuscript. MS performed the literature review and prepared the draft. PR provided critical revision of the article.

\section{FUNDING}

The processing charges cost was covered by the Maria Sklodowska-Curie National Research Institute of Oncology, Warsaw, Poland.

13. Kraybill WG, Harris J, Spiro IJ, Ettinger DS, DeLaney TF, Blum RH, et al. Phase II study of neoadjuvant chemotherapy and radiation therapy in the management of high-risk, high-grade, soft tissue sarcomas of the extremities and body wall: radiation therapy oncology group trial 9514. J Clin Oncol. (2006) 24:619-25. doi: 10.1200/JCO.2005.02.5577

14. Canter RJ, Martinez SR, Tamurian RM, Wilton M, Li C-S, Ryu J, et al. Radiographic and histologic response to neoadjuvant radiotherapy in patients with soft tissue sarcoma. Ann Surg Oncol. (2010) 17:2578-84. doi: 10.1245/s10434-010-1156-3

15. Yoon SS, Duda DG, Karl DL, Kim T-M, Kambadakone AR, Chen Y-L, et al. Phase II study of neoadjuvant bevacizumab and radiotherapy for resectable soft tissue sarcomas. Int J Radiat Oncol Biol Phys. (2011) 81:108190. doi: 10.1016/j.ijrobp.2010.07.024

16. Shah D, Borys D, Martinez SR, Li CS, Tamurian RM, Bold RJ, et al. Complete pathologic response to neoadjuvant radiotherapy is predictive of oncologic outcome in patients with soft tissue sarcoma. Anticancer Res. (2012) 32:39115. Available online at: ar.iiarjournals.org/content/32/9/3911.long

17. O'Sullivan B, Griffin AM, Dickie CI, Sharpe MB, Chung PWM, Catton $\mathrm{CN}$, et al. Phase 2 study of preoperative image-guided intensity-modulated radiation therapy to reduce wound and combined modality morbidities in lower extremity soft tissue sarcoma. Cancer. (2013) 119:1878-84. doi: $10.1002 / \mathrm{cncr} .27951$

18. Lewin J, Khamly KK, Young RJ, Mitchell C, Hicks RJ, Toner GC, et al. A phase $\mathrm{Ib} / \mathrm{II}$ translational study of sunitinib with neoadjuvant radiotherapy in softtissue sarcoma. Br J Cancer. (2014) 111:2254-61. doi: 10.1038/bjc.2014.537

19. Canter RJ, Borys D, Olusanya A, Li CS, Lee LY, Boutin RD, et al. Phase I trial of neoadjuvant conformal radiotherapy plus sorafenib for patients with locally advanced soft tissue sarcoma of the extremity. Ann Surg Oncol. (2014) 21:1616-23. doi: 10.1245/s10434-014-3543-7

20. Wang D, Zhang Q, Eisenberg BL, Kane JM, Li XA, Lucas D, et al. Significant reduction of late toxicities in patients with extremity sarcoma treated with image-guided radiation therapy to a reduced target volume: results of radiation therapy oncology group RTOG-0630 trial. J Clin Oncol. (2015) 33:2231-8. doi: 10.1200/JCO.2014.58.5828

21. Haas RLM, Gelderblom $H$, Sleijfer S, van Boven $H H$, Scholten A, Dewit L, et al. A phase I study on the combination of neoadjuvant radiotherapy plus pazopanib in patients with locally advanced soft tissue sarcoma of the extremities. Acta Oncol. (2015) 54:1195-201. doi: 10.3109/0284186X.2015.1037404

22. Jakob J, Simeonova A, Kasper B, Ronellenfitsch U, Rauch G, Wenz $\mathrm{F}$, et al. Combined sunitinib and radiation therapy for preoperative treatment of soft tissue sarcoma: results of a phase I trial of the German interdisciplinary sarcoma group (GISG-03). Radiat Oncol. (2016) 11:77. doi: 10.1186/s13014-016-0654-2

23. Temple WJ, Temple CL, Arthur K, Schachar NS, Paterson AH, Crabtree TS. Prospective cohort study of neoadjuvant treatment in conservative surgery of soft tissue sarcomas. Ann Surg Oncol. (1997) 4:586-90. doi: 10.1007/BF02305541

24. Ryan CW, Montag AG, Hosenpud JR, Samuels B, Hayden JB, Hung AY, et al. Histologic response of dose-intense chemotherapy with preoperative 
hypofractionated radiotherapy for patients with high-risk soft tissue sarcomas. Cancer. (2008) 112:2432-9. doi: 10.1002/cncr.23478

25. MacDermed DM, Miller LL, Peabody TD, Simon MA, Luu HH, Haydon RC, et al. Primary tumor necrosis predicts distant control in locally advanced soft-tissue sarcomas after preoperative concurrent chemoradiotherapy. Int J Radiat Oncol Biol Phys. (2010) 76:1147-53. doi: 10.1016/j.ijrobp.2009. 03.015

26. Meyer JM, Perlewitz KS, Hayden JB, Doung Y-C, Hung AY, Vetto JT, et al. Phase I trial of preoperative chemoradiation plus sorafenib for high-risk extremity soft tissue sarcomas with dynamic contrastenhanced MRI correlates. Clin Cancer Res. (2013) 19:6902-11. doi: 10.1158/1078-0432.CCR-13-1594

27. Koseła-Paterczyk H, Szacht M, Morysinski T, Ługowska I, Dziewirski W, Falkowski S, Zdzienicki M, et al. Preoperative hypofractionated radiotherapy in the treatment of localized soft tissue sarcomas. Eur J Surg Oncol. (2014) 40:1641-7. doi: 10.1016/j.ejso.2014. 05.016

28. Pennington JD, Eilber FC, Eilber FR, Singh AS, Reed JP, Chmielowski B, et al. Long-term outcomes with ifosfamide-based hypofractionated preoperative chemoradiotherapy for extremity soft tissue sarcomas. Am J Clin Oncol. (2018) 41:1154-61. doi: 10.1097/COC.00000000000 00443

29. Spałek M, Koseła-Paterczyk H, Borkowska A, Wagrodzki M, SzumeraCiećkiewicz A, Pałucki J, et al. Hypofractionated radiotherapy in locally advanced myxoid liposarcomas of extremities or trunk wall: results of a single arm prospective clinical trial. Int $J$ Radiation Oncol Biol Phys. (2019) 105:S63. doi: 10.1016/j.ijrobp.2019. 06.506
30. Parsai S, Lawrenz J, Kilpatrick S, Rubin B, Hymes C, Gray M, et al. Early outcomes of preoperative 5-fraction radiation therapy for soft tissue sarcoma followed by immediate surgical resection. medRxiv. (2020). doi: $10.1101 / 2020.03 .20 .20038885$

31. Kalbasi A, Kamrava M, Chu FI, Telesca D, Dams RV, Yang Y, et al. A phase 2 trial of five-day neoadjuvant radiation therapy for patients with high-risk primary soft tissue sarcoma. Clin Cancer Res. (2020) 26:clincanres.3524. doi: 10.1158/1078-0432.CCR-19-3524

32. Slump J, Bastiaannet E, Halka A, Hoekstra HJ, Ferguson PC, Wunder JS, et al. Risk factors for postoperative wound complications after extremity soft tissue sarcoma resection: a systematic review and meta-analyses. J Plast Reconstr Aesthet Surg. (2019) 72:1449-64. doi: 10.1016/j.bjps.2019.05.041

33. Dickie CI, Parent AL, Griffin AM, Fung S, Chung PWM, Catton CN, et al Bone fractures following external beam radiotherapy and limb-preservation surgery for lower extremity soft tissue sarcoma: relationship to irradiated bone length, volume, tumor location and dose. Int J Radiat Oncol Biol Phys. (2009) 75:1119-24. doi: 10.1016/j.ijrobp.2008.12.006

Conflict of Interest: The authors declare that the research was conducted in the absence of any commercial or financial relationships that could be construed as a potential conflict of interest.

Copyright $\odot 2020$ Spatek and Rutkowski. This is an open-access article distributed under the terms of the Creative Commons Attribution License (CC BY). The use, distribution or reproduction in other forums is permitted, provided the original author(s) and the copyright owner(s) are credited and that the original publication in this journal is cited, in accordance with accepted academic practice. No use distribution or reproduction is permitted which does not comply with these terms. 